\title{
Cabibbo Angle from the Nonlinear Dynamics of Quark Currents
}

\author{
Ervin Goldfain \\ Research Scholar, Ronin Institute, Montclair, New Jersey 07043 \\ Email: ervin.goldfain@ronininstitute.org
}

\begin{abstract}
Our earlier work has tentatively shown that the hierarchy of fermion masses and mixing angles follows from the universal behavior of nonlinear dynamics. In this brief sequel we survey a similar scenario in which the Cabibbo angle arises from the nonlinear dynamics of charged-current interactions.
\end{abstract}

Key words: Cabibbo angle, fermion mixing, charged current interactions, nonlinear dynamics and chaos, complex dynamics, Feigenbaum's constant.

Charged current interactions are flavor-changing processes mediated by the $W^{ \pm}$bosons and described by the Lagrangian [1]

$$
L_{C C}=\frac{g}{\sqrt{2}}\left(J_{\mu}^{+} W^{+\mu}+J_{\mu}^{-} W^{-\mu}\right)
$$

Here, the current written in the unitary gauge is given by

$$
J_{\mu}^{+}=v_{L}^{\prime} \gamma_{\mu} e_{L}^{\prime}+\bar{u}_{L}^{\prime} \gamma_{\mu} \bar{d}_{L}^{\prime}
$$

Because in the Standard Model fermion mass eigenstates do not coincide with the weak eigenstates, the weak eigenstates are rotations of the mass eigenstates via the Cabibbo 
angle $\theta_{C}$. In the simple case of only two generations, the Cabibbo rotation leads to quark mixing and takes the form

$$
\left(\begin{array}{l}
d^{\prime} \\
s^{\prime}
\end{array}\right)_{L}=\left(\begin{array}{cc}
\cos \theta_{C} & \sin \theta_{C} \\
-\sin \theta_{C} & \cos \theta_{C}
\end{array}\right)\left(\begin{array}{l}
d \\
s
\end{array}\right)_{L}
$$

The charged current transition from a $d^{\prime}$ quark to a $u$ quark by absorption of a $W^{+}$gauge boson is represented by the Lagrangian

$$
L_{C C}=\frac{g}{\sqrt{2}}\left(\begin{array}{ll}
\bar{u} & \bar{c}
\end{array}\right)_{L} \gamma^{\mu}\left(\begin{array}{cc}
\cos \theta_{C} & \sin \theta_{C} \\
-\sin \theta_{C} & \cos \theta_{C}
\end{array}\right)\left(\begin{array}{l}
d \\
s
\end{array}\right)_{L} W_{\mu}^{+}+\text {h.c. }
$$

Drawing from the onset of complex dynamics near the electroweak scale [2-3, 7-8], an attractive dynamical model of (4) may be developed starting from the framework of coupled nonlinear oscillators. The most straightforward path to building this model is through the generic theory of the damped and driven pendulum [4]. To this end, the quark pairs entering (4) may be regarded as two independent oscillators coupled through the steady exchange of the $W^{+}$boson. In what follows we assume that

a) the driving frequency $\omega$ stands for the frequency of the $W^{+}$boson,

b) the damping coefficient $\beta>0$ embodies the combined effect of unbalanced quantum fluctuations that fail to cancel out exactly at the scale of the $W^{+}$boson. These effects are absent in perturbative Quantum Field Theory but show up as signature of non-equilibrium dynamics on or above the electroweak scale [2, 9-10].

From these premises, one is led to the equation

$$
\alpha \ddot{\theta}+\beta \dot{\theta}+\gamma \sin \theta=A+K \cos \omega t, \quad \beta>0
$$


Here,

$$
\theta=\left(\begin{array}{l}
\theta_{1}=\theta_{\bar{u} \bar{c}} \\
\theta_{2}=\theta_{d s}
\end{array}\right)
$$

represents the two-degree of freedom oscillator angle and $\alpha, \beta, \gamma, A, K$ are constant parameters of the model. One successful application of (5) is the study of the Josephson junction in the theory of superconductivity [4]. Experimental analysis shows that the Feigenbaum constant describing the chaotic behavior of the Josephson junction falls in the range [5]

$$
\bar{\delta}=4.4 \pm 0.3
$$

Appealing to [3] leads to the conclusion that the average value of the Cabibbo angle is given by

$$
\lambda_{C}^{a v}=\bar{\delta}^{-1}=0.2273
$$

in close agreement with current testing data [6].

\section{References}

1. Cheng, T. P., and Li L. F., "Gauge theory of elementary particle physics”, Oxford Univ. Press, 1989.

2. Available at the following site:

https://www.researchgate.net/publication/344227402_Complex_Dynamics_and_Fou ndational_Physics 
3. Available at the following site:

https://www.researchgate.net/publication/344036923 Mass Hierarchy and Mixing Matrices from Universal Bifurcations

4. McCauley, J. L., “Chaos, Dynamics and Fractals”, Cambridge Univ. Press, 1994.

5. Peitgen, H. O., et al., "Chaos and Fractals", Springer-Verlag, 1992.

6. https://cds.cern.ch/record/688971/files/p23.pdf

7. Available at the following site:

https://www.researchgate.net/publication/228383180 Chaos in Quantum Chromod ynamics and the Hadron Spectrum

8. Available at the following site:

https://www.sciencedirect.com/science/article/abs/pii/S1007570408000099?via\%3Di $\underline{\text { hub }}$

9. http://www.ejtp.com/articles/ejtpv7i24p219.pdf

10. http://www.novapublishers.org/catalog/product info.php?products id=35813 